\title{
What drives individual differences in statistical learning? The role of perceptual fluency and familiarity
}

\author{
Andrew Perfors \\ School of Psychological Sciences \\ University of Melbourne \\ ARC Centre of Excellence for the Dynamics of Language \\ Evan Kidd \\ Max Planck Institute for Psycholinguistics \\ Research School of Psychology \\ The Australian National University \\ ARC Centre of Excellence for the Dynamics of Language
}




\begin{abstract}
Humans have the ability to learn surprisingly complicated statistical information in a variety of modalities and situations, often based on relatively little input. These statistical learning (SL) skills appear to underlie many kinds of learning, but despite their ubiquity, we still do not fully understand precisely what SL is and what individual differences on SL tasks reflect. Here we present experimental work suggesting that at least some individual differences arise from variation in perceptual fluency — the ability to rapidly or efficiently code and remember the stimuli that statistical learning occurs over - and that perceptual fluency is driven at least in part by stimulus familiarity: performance on a standard SL task varies substantially within the same (visual) modality as a function of whether the stimuli involved are familiar or not, independent of stimulus complexity. Moreover, we find that test-retest correlations of performance in a statistical learning task using stimuli of the same level of familiarity (but distinct items) are stronger than correlations across the same task with stimuli of different levels of familiarity. Finally, we demonstrate that statistical learning performance is predicted by an independent measure of stimulus-specific perceptual fluency that contains no statistical learning component at all. Our results suggest that a key component of statistical learning performance may be related to stimulus-specific perceptual processing and familiarity.
\end{abstract}




\section{Introduction}

Statistical learning (SL) refers to the ability to adapt to and learn from the probabilistic structure of the environment. This ability is phylogenetically old (Hauser, Newport, \& Aslin, 2001) and appears to be operational quite early in development (Teinonen, Fellman, Näätänen, Alku, \& Huotilainen, 2009; Bulf, Johnson, \& Valenza, 2011). It occurs across multiple modalities and across a wide variety of types of stimuli (Saffran, Aslin, \& Newport, 1996; Fiser \& Aslin, 2002; Kirkham, Slemmer, \& Johnson, 2002; Brady \& Oliva, 2008; Gebhart, Newport, \& Aslin, 2009; Krogh, Vlach, \& Johnson, 2013; Buchsbaum, Griffiths, Plunkett, Gopnik, \& Baldwin, 2015). Moreover, recent evidence suggests that individual differences in statistical learning abilities appear to be stable and reliable (Isbilen, McCauley, Kidd, \& Christiansen, 2017, 2020; Siegelman, Bogaerts, Elazar, Arciuli, \& Frost, 2018). Taken together, these findings appear to indicate that statistical learning is a flexible and broadly applicable capacity for extracting and using the regularities in the world.

The broad applicability of statistical learning meant that, for some time, the field implicitly adopted a "unitary" conception of it, assuming it to be a domain-general mechanism that operated similarly across different modalities. However, as Frost, Armstrong, and Christiansen (2019) note, a unitary conception of SL as reflecting modality-independent abstract computations is inconsistent with several lines of research. Notably, behavioral data suggests a large degree of stimulus specificity. For instance, Conway and Christiansen (2006) reported that participants were most capable of acquiring two artificial grammars simultaneously when those grammars were presented in different modalities, a result which is difficult to accommodate under a single mechanism view. Complementary data come from Siegelman and Frost (2015), who found no association between performance on tasks measuring visual and auditory SL for linguistic stimuli. Interestingly, Siegelman et al. (2018) reported a significant association between visual SL and SL for non-linguistic sounds (e.g., a siren, dog barking), but once again not with linguistic stimuli. They interpreted the pattern of results to reflect linguistic entrenchment. That is, while participants came to their studies without prior knowledge and expectations regarding how visual shapes and non-linguistic sounds were distributed, their life-long experience with language led to expectations regarding how syllables patterned in their language to form words. Consistent with this assertion, they found that independent ratings of the 'wordiness' of their novel linguistic stimuli positively predicted participant's performance, such that more Hebrew-like words (the native language of their participants) were more easily acquired.

These data suggest that both stimulus specificity and domain-independence play a role in SL. Moreover, in the case of Siegelman et al. (2018), they suggest that prior learning constitutes an important yet understudied component of the process. As a result, statistical learning has more recently been conceptualised as a multi-component process (Arciuli, 2017; Frost et al., 2019; Siegelman, Bogaerts, \& Frost, 2017), grounded in basic perception and cognition ((Frank, Goldwater, Griffiths, \& Tenenbaum, 2010; Christiansen, 2019; Isbilen et al., 2020). However, the specifics of those components are far from clear, as Siegelman et al. (2017) note: "in contrast with general intelligence ... the dimensions of SL as an individual ability are yet to be empirically established." (p. 5). In this paper we build upon previous theoretical and empirical work to explore the role of perceptual fluency and stimulus familiarity in visual statistical learning.

Frost, Armstrong, Siegelman, and Christiansen (2015) suggested that variance in performance on statistical learning tasks comes from two main sources - not just (i) variability in the efficiency with which domain-general learning mechanisms detect the statistical properties of the input stream, but also (ii) variability in the efficiency of perceptual encoding within each modality. 
Under this theory, SL across modalities depends on similar domain-general computations, but SL tasks are typically uncorrelated because modality-dependent processes significantly influence how distributions are learned. One test of this theory came from presenting participants with nine different visual SL tasks that factorially manipulated speed of presentation $(200 \mathrm{~ms}, 600 \mathrm{~ms}$, or $1000 \mathrm{~s})$ and probabilistic structure (within-pair TPs were 0.6, 0.8, or 1.0) (Bogaerts, Siegelman, \& Frost, 2016). As predicted, both variables affected learning, with higher performance observed at slower exposure rates and for high TPs. Participants who benefited most from the longer inspection times were also better at learning the underlying statistical structure (and vice-versa). This suggests that an individual's perceptual fluency may afford a significant advantage in statistical learning, and is consistent with the observation that at least one measure of statistical learning has been found to be associated with psychometric measures of processing speed (Kaufman et al., 2010).

But what makes a person have high perceptual fluency on a given set of stimuli? One possibility, as Frost et al. (2015) theorised, is that it is entirely modality specific: the brain processes stimuli differently in different modalities. Another possibility is that fluency increases with the amount of time available for encoding (Turk-Browne, Junge, \& Scholl, 2005; Arciuli \& Simpson, 2011; Bogaerts et al., 2016). These factors are well-studied and are likely to play an important role.

However, there is yet another, less well-studied possible factor: stimulus familiarity. Because familiar items are easier to quickly encode and accurately remember (Jackson \& Raymond, 2008; Xie \& Zhang, 2017), familiarity might free up cognitive capacity to devote to other tasks (like statistical learning). As a result, one might expect SL performance to be higher when the items involved are familiar rather than unfamiliar, even if the underlying statistical regularities are the same in both cases. Moreover, to the extent that perceptual fluency reflects familiarity rather than modality-specific skills, one might expect that correlations between SL tasks would be affected by the perceptual similarity of the stimuli even when the modality is held constant. Finally, if statistical learning is affected by perceptual fluency, one might expect SL performance to be predicted by a performance on a purely perceptual stimulus-specific task with no statistical learning component at all. We test all of these predictions in this work.

It is worth noting that although our hypothesis is that individual differences in statistical learning are moderated by perceptual fluency - which is shaped by familiarity - our work is distinct from other research that also suggests that prior experience plays a role in statistical learning of linguistic information (e.g., Gebhart et al., 2009; Endress \& Mehler, 2009; Perruchet \& PoulinCharronnat, 2012; Siegelman et al., 2018). Our focus here is on the role that familiarity may play in making individual stimulus items easier to remember and parse; we thus predict that previous exposure should facilitate statistical learning in any modality, not interfere with statistical learning in the linguistic one. The focus of previous work is on the role that prior experience plays in learning statistical regularities between items; it suggests that previous linguistic exposure may affect SL due to interference and entrenchment, and the focus is not on the items so much as the transition probabilities between them. Our approach complements this other research because both focus on the role of prior experience in shaping statistical learning; it is distinct because it has a different mechanism and makes different predictions.

\section{Experiment 1: Method}

This research tests the hypothesis that individual differences in statistical learning are mediated by individual differences in perceptual fluency, which is itself shaped by stimulus familiarity. We investigated this by having the same individuals participate in two different visual SL tasks on 
two different days, as shown in Figure 1. On each day people were randomly assigned to one of two conditions (FAMILIAR and UNFAMILIAR) in which the SL task was the same and the only difference was whether the stimuli involved were novel to the participant or not. Thus, some people saw stimuli with the same level of novelty on each day (although distinct items each time) while others saw the FAMILIAR stimuli on one day and the UNFAMILIAR ones on another. If stimulus familiarity plays an important mediating role, we would expect that SL performance is higher when the stimuli are FAMILIAR. Additionally, we also expected that there would be a higher test-retest correlation across sessions when the stimulus novelty is the same, since all of the processes involved in visual statistical learning - perceptual encoding of stimuli as well as the computation of TPs between them - are the same.

In addition to the statistical learning tasks that occurred during both experimental sessions, Session 2 also presented participants with two measures of perceptual fluency (PF) for the stimuli they had previously seen (the first PF task used the stimuli from Session 1, and the second used the stimuli from Session 2). The PF task was designed to measure the speed with which these particular stimuli could be parsed and encoded. If perceptual fluency plays an important role in statistical learning, we would expect that PF task performance would be correlated with SL task performance.

SAME FAMILIARITY
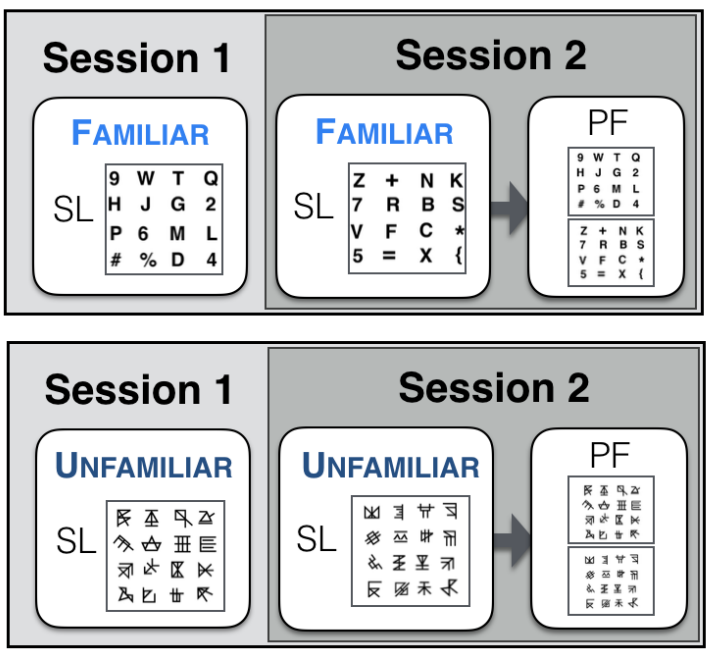

DIFFERENT FAMILIARITY
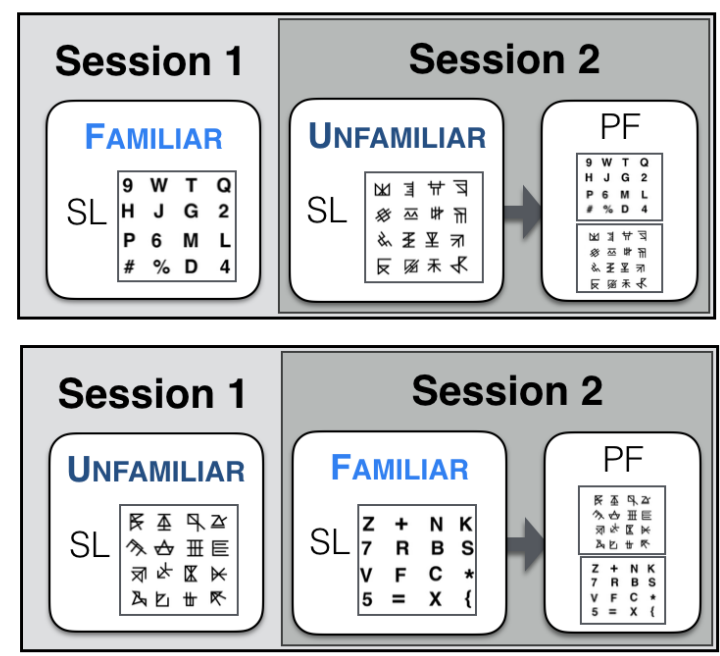

Figure 1. Overview of experiment structure. Each participant was tested in two sessions approximately one week apart. In the first session, people performed an embedded triplet visual statistical learning (SL) task using either FAMILIAR or UNFAMILIAR stimuli. In the second session, the same participants completed another SL task with new stimuli that either matched or did not match in familiarity (although in all cases the specific stimulus items were different). Following the SL task, each person performed two consecutive tasks that measured their perceptual fluency (PF) on the two stimulus sets they had seen.

\section{Participants}

For Session 1, 160 participants were recruited from Amazon Mechanical Turk for the 15minute task, for which they were paid \$3.50USD. Of these, 14 were excluded for failing the at- 
tention check (described below). The remaining 146 were invited to return for a second 20-minute session a week later, for which they were paid \$4USD. ${ }^{1} 135$ returned, three of whom failed the attention check. All analyses focus on the remaining 132 participants, 73 (55.3\%) of whom were male and 129 (97.7\%) were from the US. Ages ranged from 20 to 69 (mean 36.1). At each session participants were randomly assigned to either FAMILIAR or UNFAMILIAR stimulus sets of 16 items each. This resulted in 53 people who saw the SAME stimulus complexity each time (17 FAMILIAR, 36 UNFAMILIAR) and 79 people who saw a DIFFERENT stimulus complexity each time (44 saw FAMILIAR first, 35 saw UNFAMILIAR first).

\section{Materials}

As shown in Figure 2, the UNFAMILIAR stimuli were created by combining between four and six straight lines, resulting in novel shapes that were perceptually discriminable but effortful to parse and remember. The FAMILIAR stimuli consisted of letters and symbols. We chose these because they are highly over-learned and very familiar to any literate English speaker. In order to minimise the potential for "chunking" letter combinations into existing words, no vowels or vowellike symbols like @ or \& were included. Moreover, all participants in all conditions saw a different random combination of the stimuli into triples, as described below.

Session 1

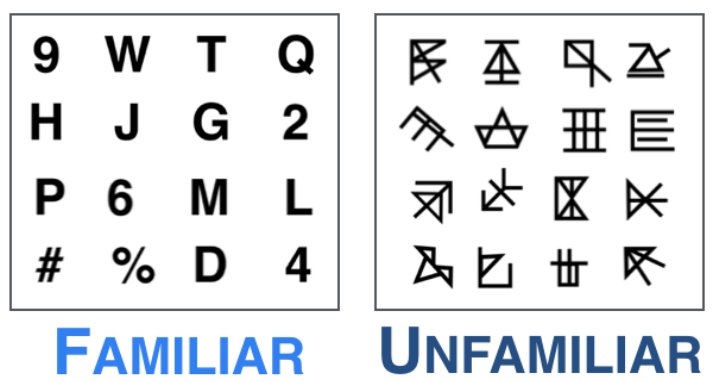

Session 2

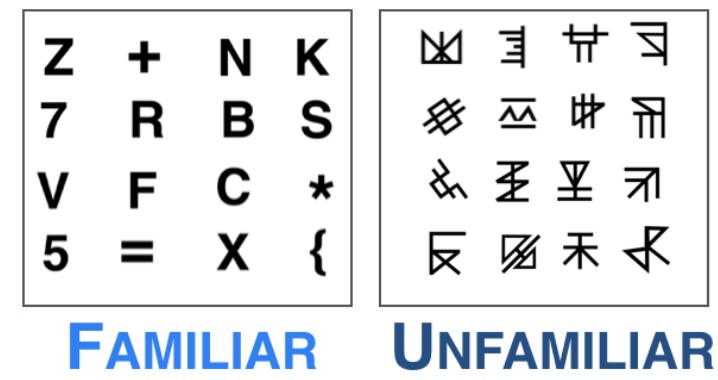

Figure 2. Experiment 1 stimuli. At each session, participants saw 16 stimulus items that were either FAMILIAR or UnFAMILIAR, depending on randomly-assigned condition. Although some people saw stimuli of similar familiarity in both sessions, no single item occurred both times.

\section{Procedure}

Statistical Learning (SL) task. The statistical learning task in both sessions was nearly identical to Siegelman et al. (2017), which was designed to have good psychometric properties including reasonable test-retest reliability and the ability to differentiate between individuals. Besides the specific stimuli used, the only difference between our task and theirs is that we included an attention check by embedding four or five English words in the training sequence. (In Session 1 these words were train, boy, lion, and walk. In Session 2 they were boat, lady, koala, tree, and doll.) Before training, participants were told that they should simply watch the sequence, but that to ensure attention we had included a few English words amongst the symbols; they were not informed

\footnotetext{
${ }^{1}$ https://github.com/perfors/vsl/ contains stimuli, experiment code, data, and analyses for all experiments.
} 
about how many there would be. They were told to write down any English words as they occurred and report them in a text box at the end of training. People who got fewer than three correct in either session were excluded from all analyses.

Following Siegelman et al. (2017), the statistical learning task itself consisted of 16 items combined into 8 triplets, each repeated 24 times in random order. The task is divided into two phases: (i) a familiarzation phase, and (ii) a test phase. During the familiarization phase each item appeared alone at the centre of the participant's screen for $800 \mathrm{~ms}$ with a $200 \mathrm{~ms}$ inter-stimulus interval. The eight triplets were designed so that four were easy, defined by transition probabilities (TPs) of 1.0 between each item $\left(x_{5}-x_{6}-x_{7}, x_{8}-x_{9}-x_{10}, x_{11}-x_{12}-x_{13}, x_{14}-x_{15}-x_{16}\right)$ while the other four were harder, defined by TPs of 0.33 between items $\left(x_{1}-x_{2}-x_{3}, x_{2}-x_{1}-x_{4}, x_{4}-x_{3}-x_{1}, x_{3}-x_{4}-x_{2}\right)$. The mapping of each image to each item, as well as the order of the triplets and the location of the embedded English words, was randomised for each participant at each session (thus the TP between triplets was $1 / 8=0.125)$. The presentation of triplets was constrained so that triplets were never immediately repeated. The familiarization phase lasted 10 minutes.

The test phase immediately followed. We administered the exact same 42 -item test as in Siegelman et al. (2017). In contrast to many common SL tasks, which typically use two-alternative forced choice (2AFC) as a sole measure of learning, the task uses an array of different method to measure learning. These were: (i) $162 \mathrm{AFC}$ trials, where participants compared an attested triplet to an unattested foil, (ii) $84 \mathrm{AFC}$ trials, where participants compared triplets to three foils, (iii) $62 \mathrm{AFC}$ trails where participants compared attested pairs (i.e., pairs of shapes that had bigrams of either .33 or 1.0) to pairs with lower bigrams), (iv) $44 \mathrm{AFC}$ 'pair' trials, and (v) 8 pattern completion trials, where participants where required to complete 4 triplets patterns and 4 pair patterns by selecting the correct shape. For more information see Siegelman et al. (2017).

For each participant we calculated an overall statistical learning score, SLscore, which reflects the proportion of the 42 test items that they got correct. In order to evaluate whether our participants found the same items to be difficult as the participants in Siegelman et al. (2017), we calculated the correlation for performance on each item between our participants and theirs. It was significant in both conditions (FAMILIAR: $r=0.8, p<0.0001$; UNFAMILIAR: $r=0.68, p<0.0001$ ), suggesting that our participants were approaching the task similarly to theirs.

Perceptual Fluency (PF) task. The Perceptual Fluency (PF) task, illustrated in Figure 3, is a novel measure we designed to capture the facility with which people could encode and recall the specific stimuli in the experiment. Inspired in part by the Inspection Time literature (see O'Connor $\&$ Burns, 2003, for an overview), each of the 48 trials in the task involves flashing an item onto the screen for a small amount of time (250ms on the first trial) followed by a masking stimulus (always presented for $200 \mathrm{~ms}$ ). The task is adaptive: if the participant successfully identifies the item, the target on the next trial flashes more quickly (with a duration of $15 \mathrm{~ms}$ less); if they do not, it flashes slower (with a duration of $15 \mathrm{~ms}$ more). Over the course of the task, participants who can achieve similar accuracy for targets displayed for a shorter duration have higher perceptual fluency for those stimuli. This is reflected in their PFscore, which consists of the mean target latency over all trials; a lower PFscore thus reflects improved perceptual fluency. For our analyses we removed a single outlier that was more than 4SD from the mean of PFscore. ${ }^{2}$

Each of the 16 items in the relevant stimulus set was the target three times over the course

\footnotetext{
${ }^{2}$ Results are qualitatively the same if no outliers are removed or if outliers at 2SD or 3SD are removed instead. We opted to remove at 4SD because inspection of the data suggested that this particular data point appeared to be a highleverage outlier with a very long relative latency, suggesting that fatigue or failure to engage was driving it.
} 
of the 48 trials. Masks and distractors were selected from the same stimulus set and completely randomised for each person, as was the order of trials.

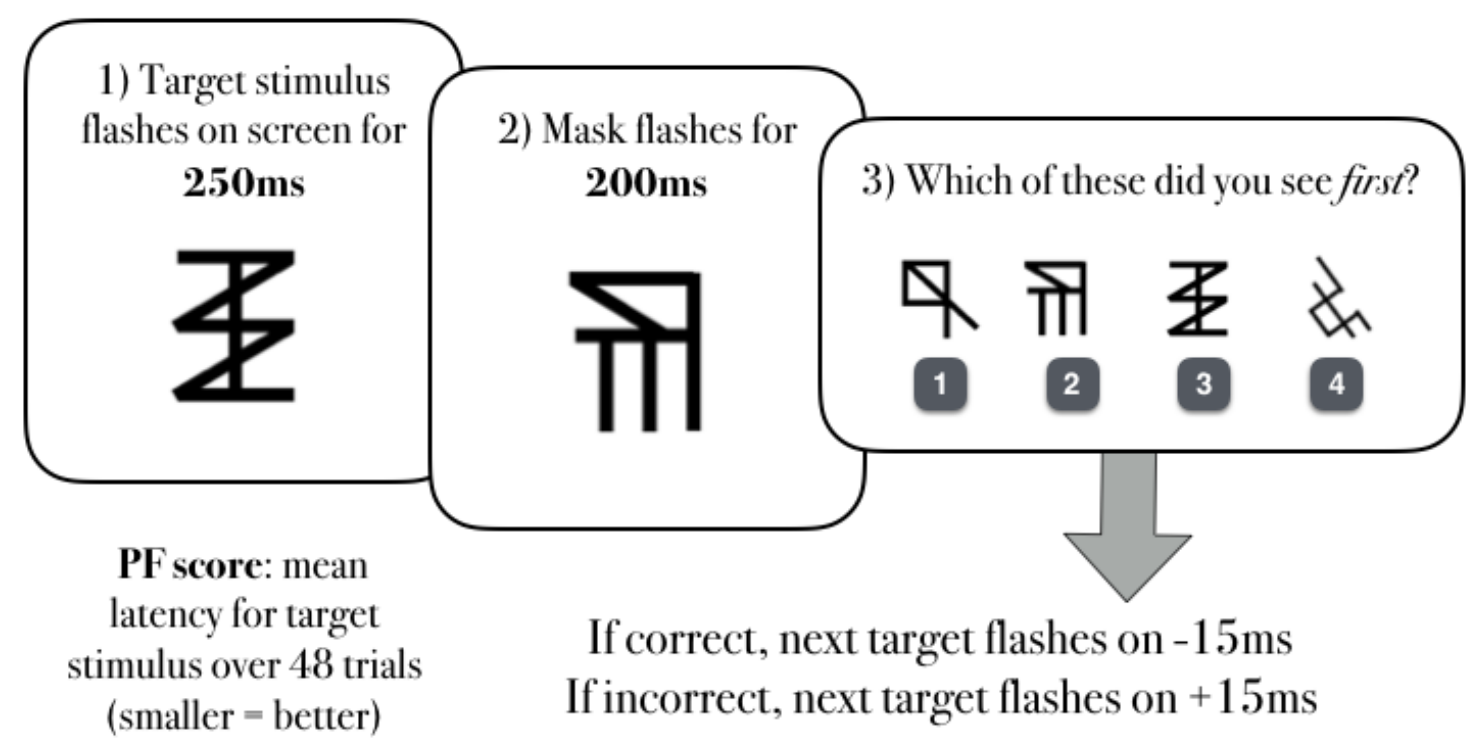

Figure 3. Perceptual fluency task. During each trial of the PF task, a target stimulus is flashed briefly onscreen, followed by a masking stimulus for $200 \mathrm{~ms}$. Participants are then presented with four items (the target, the mask, and two others randomly selected from the stimulus set). If they successfully identify the target, on the next trial the duration of target presentation decreases by $15 \mathrm{~ms}$; if not, it increases by $15 \mathrm{~ms}$. The perceptual fluency score (PFscore) reflects the mean target latency over all 48 trials. Smaller PFscore indicates better perceptual fluency, because it indicates that the person can attain similar accuracy based on a shorter target presentation time.

\section{Experiment 1: Results}

The overall hypothesis being tested in this paper is that perceptual fluency plays a mediating role in performance on statistical learning tasks. This hypothesis yields several testable predictions. First, it predicts that people will show different levels of performance on statistical learning in tasks that differ only on familiarity, for which the SL component is identical. Second, it predicts that correlations between SL performance at two different time points should be greater if the stimuli are of similar familiarity than if they are not (even though all are within the same modality). Third, it predicts that statistical learning performance should be correlated with perceptual fluency, even though no statistical learning is involved in the perceptual fluency task at all. We consider each of these predictions in turn.

\section{Is performance better on Familiar stimuli?}

Do people improve when the stimuli involved are FAMILIAR rather than UNFAMILIAR? We ask this question separately for both SL and PF tasks. Appendix A contains more detail. 
Statistical learning. Accuracy on the statistical learning tasks, as measured by SLscore, does appear to span the range of individual differences, ranging between $26.2 \%$ to $97.6 \%$ (mean: $59.6 \%$ ) in the FAMILIAR condition and $28.6 \%$ to $92.9 \%$ (mean: $52.7 \%$ ) in the UNFAMILIAR condition. In both conditions, performance is significantly above chance, which Siegelman et al. (2017) calculate as $40 \%$ (FAMILIAR: $t(112)=11.45, p<0.0001, d=1.08$; UNFAMILIAR: $t(150)=$ $10.69, p<0.0001, d=0.87)$. Moreover, the difference in accuracy between FAMILIAR and UNFAMILIAR conditions, shown in Figure 4(a), is significant $(t(211)=3.24, p=0.0014, d=0.42$ ).

Perceptual fluency. As Figure 4(b) reveals, perceptual fluency was also significantly improved, as reflected in a lower PFscore, when the stimuli were FAMILIAR than when they were UNFAMILIAR $(t(240.1)=-3.46, p=0.0006, d=0.43)$. This is reassuring both because it helps to support the notion that PFscore is indeed measuring something about perceptual fluency, and because it suggests that participants were behaving sensibly on the PF task in general.

(a) Statistical learning

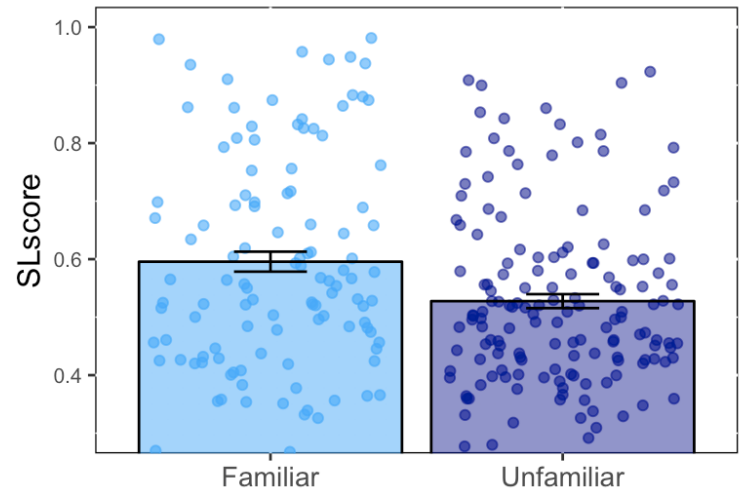

(b) Perceptual fluency

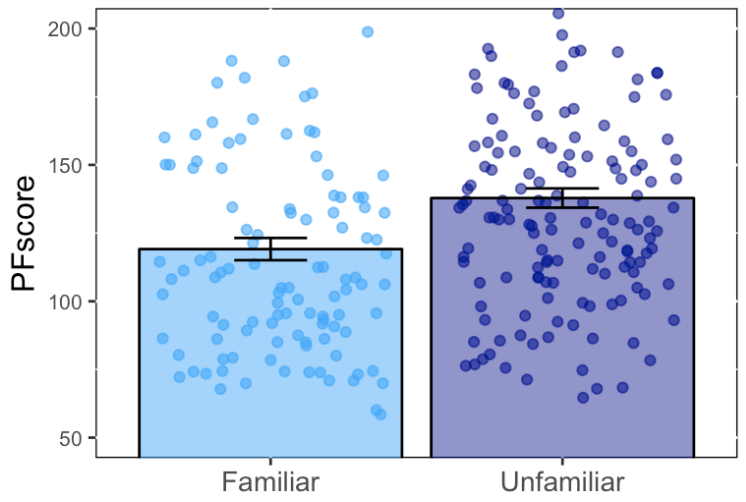

Figure 4. Performance as a function of stimulus complexity. (a) In the statistical learning task, accuracy (SLscore) was significantly higher for FAMILIAR stimuli than for UNFAMILIAR stimuli, though both were far above chance at approximately $40 \%$. (b) In the perceptual fluency task, mean latency of the target stimulus (PFscore) was significantly lower for FAMILIAR stimuli, indicating that participants were better at quickly encoding and remembering the FAMILIAR stimuli than the UNFAMILIAR ones.

\section{Are SL correlations higher between stimuli of the same familiarity?}

If perceptual fluency mediates performance on statistical learning tasks, then we should expect that test-retest correlations between SL performance on two different sessions should be reasonably high when the stimuli are of the same familiarity (FAMILIAR-FAMILIAR or UNFAMILIARUNFAMILIAR), even though no individual items are repeated. Conversely, we should expect lower correlations if they are different at different sessions (FAMILIAR-UNFAMILIAR or UNFAMILIARFAMILIAR), even though the modality is the same. We evaluate this prediction in Figure 5. It is clear that the test-retest correlation between SAME familiarity versions was higher $(r=0.7, p<0.0001)$ than between DIFFERENT versions $(r=0.43, p<0.0001)$. The difference between these correlations is itself significant using a Fisher r-to-z transformation $(z=2.26, p=0.024)$. 

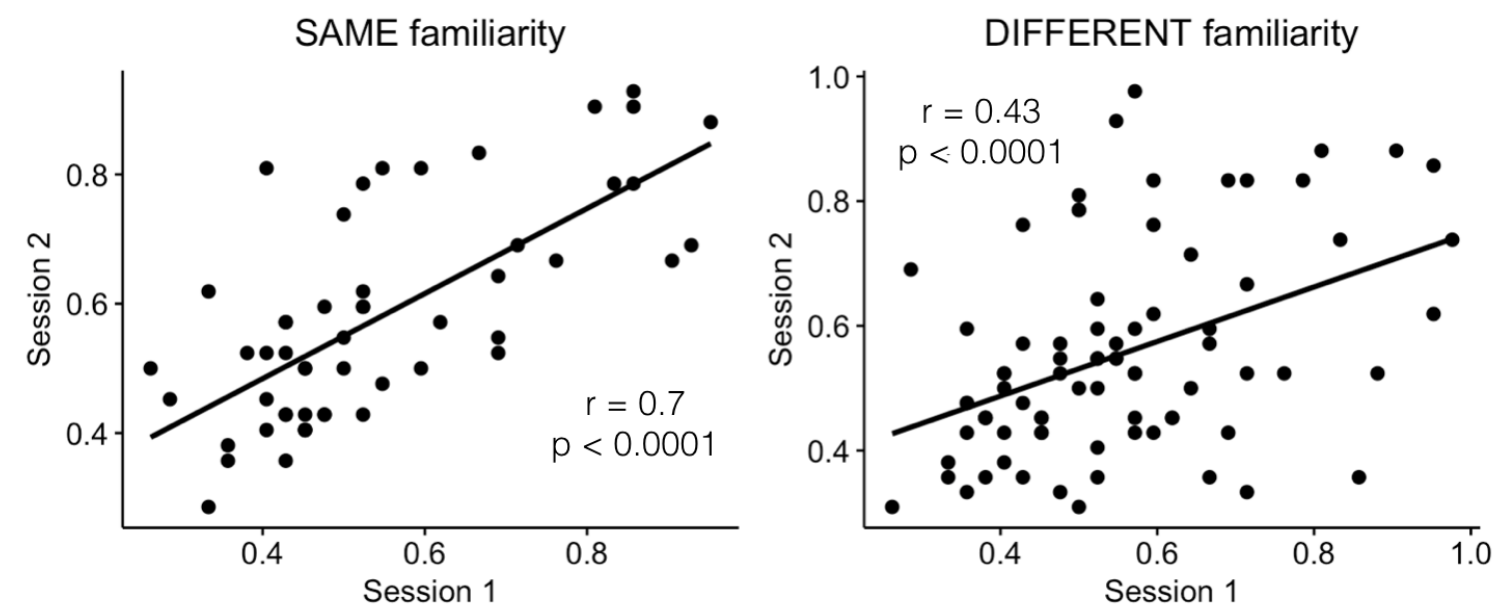

Figure 5. Test-retest correlations in SLscore as a function of stimulus complexity. The left panel shows the correlation between SLscore on Session 1 and Session 2 for those participants who performed the statistical learning task using stimuli of equal complexity each time (either FAMILIARFAMILIAR or UNFAMILIAR-UNFAMILIAR). The right panel shows the same correlation but for participants who saw stimuli of different complexity each time (either FAMILIAR-UNFAMILIAR or UNFAMILIAR-FAMILIAR). Correlations were significantly higher when the stimuli were of the same complexity, despite the fact that all were in the same (visual) modality and none of the specific items were repeated between sessions.

\section{Does perceptual fluency predict performance in the statistical learning task?}

A final prediction of the hypothesis that perceptual fluency mediates statistical learning performance is that higher perceptual fluency (as measured by a lower PFscore) should be associated with higher statistical learning (as measured by a higher SLscore). The correlation between perceptual fluency and statistical learning accuracy within the same person was indeed significant both across the same stimuli $(r=-0.37, p<0.0001)$ and different stimuli $(r=-0.28, p<0.0001)$; the difference between these correlations is not itself significant $(z=-0.76, p=0.45)$.

\section{Experiment 2: Method}

The results thus far suggest that perceptual fluency influences statistical learning, and that people have higher perceptual fluency for familiar items. Statistical learning performance is higher when the items involved are overlearned and highly familiar; test-retest correlations between SL tasks are higher when the stimuli on both days are of the same level of familiarity; and performance on a test of perceptual fluency is both higher for familiar than unfamiliar items and correlated with statistical learning performance.

However, an open question remains: to what extent do our results reflect familiarity, and to what extent do they reflect complexity? Perhaps participants performed worse on the UNFAMILIAR stimuli not because they were novel, but because they were more visually complex. After all, each item contained on average more distinct lines as well as fewer other shapes than letters do; they thus may have required finer-grained parsing than the FAMILIAR stimuli. The possibility that 
complexity rather than familiarity is driving these effects is especially interesting in light of the fact that complexity is thought to affect working memory capacity (Alvarez \& Cavanagh, 2004) and retrieval (Hofmeister, 2011).

We tested this possibility in Experiment 2, which presents another group of participants with stimuli that were designed to be simpler than the UNFAMILIAR stimuli in Experiment 1, while still being unfamiliar (we call them UNFSIMPLE). If complexity was driving the results in Experiment 1, we would expect SL performance using the UNFSIMPLE stimuli to be similar to the FAMILIAR stimuli, since both are relatively simple; if not, we would expect performance on the UNFSIMPLE stimuli to be similar to the UNFAMILIAR ones, since both are unfamiliar.

\section{Participants}

80 participants were recruited from Amazon Mechanical Turk for the 15-minute task, for which they were paid \$3.50USD. Of these, 6 were excluded for failing the attention check, which was identical to the one in Experiment 1. All analyses focus on the remaining 74 participants, $46(62.2 \%)$ of whom were male and $70(94.6 \%)$ were from the US. Ages ranged from 21 to 62 (mean 36.4). All participants were assigned to a single condition involving the UNFSIMPLE stimuli, described below. None of the participants were in Experiment 1.

\section{Materials}

Our goal in Experiment 2 was to design stimuli that were not as visually complex as the UNFAMILIAR stimuli in Experiment 1. Defining visual complexity is a difficult task and to our knowledge there is no agreed-upon answer (see, e.g., Luck \& Vogel, 1997; Alvarez \& Cavanagh, 2004); we thus followed the general principle that simpler items are those with both fewer and more distinguishable features. The UNFSIMPLE stimuli were therefore composed of fewer features than the UNFAMILIAR ones (between two to four rather than four to six) and those features were more distinguishable (lines, arcs, and circles, not just lines). They are shown in Figure 6.

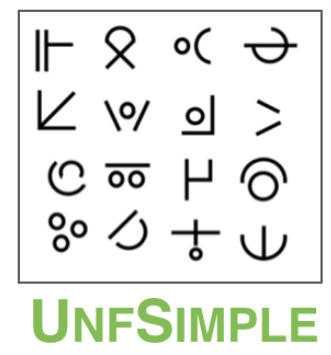

Figure 6. Experiment 2 stimuli. All participants saw 16 unfamiliar UNFSIMPLE stimulus items that were designed to be visually less complex than the UNFAMILIAR ones in Experiment 1, with fewer and more distinguishable features.

\section{Procedure}

The procedure was identical to the statistical learning task in Session 1 of Experiment 1, except with UNFSIMPLE stimuli instead. 


\section{Experiment 2: Results}

As in the previous experiment, accuracy on the statistical learning task (SLscore) using the UNFSIMPLE spans the range of individual differences, with a mean of $50.5 \%$, a minimum of $26.2 \%$, and a maximum of $95.2 \%$. Performance was significantly above the chance level of $40 \%(t(73)=6.2, p<0.0001, d=0.73)$ and there was once again a high correlation between the item-level accuracy of the participants of Siegelman et al. (2017) and ours $(r=0.66, p<0.0001)$.

Most importantly, as shown in Figure 7, overall statistical learning performance on the UNFSimPLE stimuli was more similar to performance on the UNFAMILIAR stimuli than the FAMILIAR stimuli. In order to make the most appropriate comparison, we performed a one-way ANOVA on SLscore between UNFSIMPLE and the Session 1 SL task performance in the FAMILIAR and UNFAMILIAR conditions (results were not qualitatively different if instead we used the SL scores from both sessions). There was a significant effect of condition $\left(F(2,203)=4.2743, p=0.0152, \eta^{2}=\right.$ 0.04). Follow-up t-tests indicated that the difference between FAMILIAR and UNFSIMPLE was significant $(t(113.8)=2.68, p=0.0085, d=0.47)$ but not the difference between UNFAMILIAR and UNFSIMPLE $(t(142.1)=-0.63, p=0.5271, d=0.11)$. In other words, people performed more similarly to the complex and unfamiliar stimuli than to the familiar ones. This suggests that familiarity rather than complexity underlay the differences in perceptual fluency and concomitant increase in SL performance observed in Experiment 1.

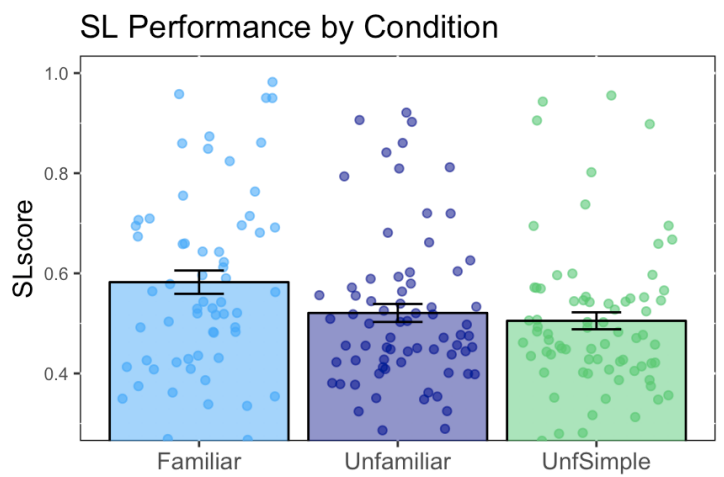

Figure 7. Experiment 2: SL performance by condition. Accuracy on the SL task (SLscore) was significantly higher for FAMILIAR stimuli than for either the unfamiliar complex stimuli (UNFAMILIAR) or the unfamiliar simple stimuli (UNFSIMPLE), suggesting that it is familiarity rather than complexity that improves statistical learning performance.

\section{Experiment 3: Method}

The results from Experiments 1 and 2 are suggestive, but it is difficult to fully measure or control for stimulus complexity. Accordingly, it is possible that the UNFSIMPLE stimuli were not actually simpler or easier to process, which would mean that - Experiment 2 notwithstanding - these effects might have been driven by complexity rather than familiarity. This would still be interesting, but would have very different implications for the nature of the mechanisms and processing that underlies statistical learning. In addition, it would be reassuring to replicate these results on a different population of participants. 
Motivated by these considerations, we conducted a third pre-registered experiment ${ }^{3}$ designed to disentangle complexity and familiarity. We dis this by holding complexity constant and testing different groups of participants who vary in their familiarity with the stimuli. Specifically, we used two sets of stimuli, Chinese characters and the unfamiliar stimuli from Study 1, and tested both native Chinese speakers, for whom the Chinese characters were over-learned and highly familiar, and English speakers, who had little or no experience with Chinese. If familiarity were driving the effect, one would expect that the Chinese participants would show better statistical learning and higher perceptual fluency for the Chinese stimuli, but that all participants would perform equally on completely novel unfamiliar stimuli.

\section{Participants}

206 undergraduates enrolled in first-year psychology at the University of Melbourne participated in this 35-45 minute experiment in order to receive course credit. ${ }^{4}$ Of these, 29 were excluded for failing the pre-registered attention check, which was identical to the one in Experiment 1 and consisted of correctly reporting the English words that had been embedded in the training sequences of the statistical learning tasks. All analyses focus on the remaining 177 participants, $48(27.1 \%)$ of whom were male. Ages ranged from 17 to 27 (mean 19.4).

All participants took part in the exact same experiment, described below, but were classified into either the CHINESE or ENGLISH group according to the following pre-registered criteria. First they took a short four-question quiz in which they were asked to choose the meaning of four different Chinese characters (for questions see Appendix B). Participants who scored less than three correct were automatically assigned to the ENGLISH group. Of those who got three or more correct, those who did not rate themselves as native speakers of Chinese were also put into the ENGLISH group. This left 61 participants in the Chinese group and 116 in the English group. Since all came from the same undergraduate cohort, the groups were matched on education level, and did not differ in age $(t(157.37)=0.39, p=0.694)$.

\section{Materials}

As described below, each participant in Experiment 3 took part in the same two statistical learning and perceptual fluency tasks. In one, they saw the same UNFAMILIAR stimuli as in Experiment 1. In the other, the stimuli were common Chinese characters, as shown in Figure 8. The characters varied in visual complexity (as measured by number of strokes) and pronunciation (based on initial phoneme and tone) and all corresponded to simple nouns. Appendix B contains the full list of characters with their translation and pronunciation.

\section{Procedure}

The procedure for this experiment was similar to Experiment 1 but had a few important differences resulting from several considerations. First, in order to recruit from the undergraduate

\footnotetext{
${ }^{3}$ The preregistration can be found at http: //aspredicted. org/blind.php? $\mathrm{x}=\mathrm{r} 76 \mathrm{fb} 4$.

${ }^{4}$ We preregistered running 160 participants, expecting more difficulty in recruiting than we had, and were encouraged to run more than 160 in order to ensure that all students were able to earn sufficient course credit. All analyses reported here were performed only once, on the full dataset, but in order to ensure that our results were not due to a larger sample size than anticipated we also ran all analyses on the first 160 people in the dataset, with identical results; see https://github.com/perfors/vsl/for details of these analyses.
} 


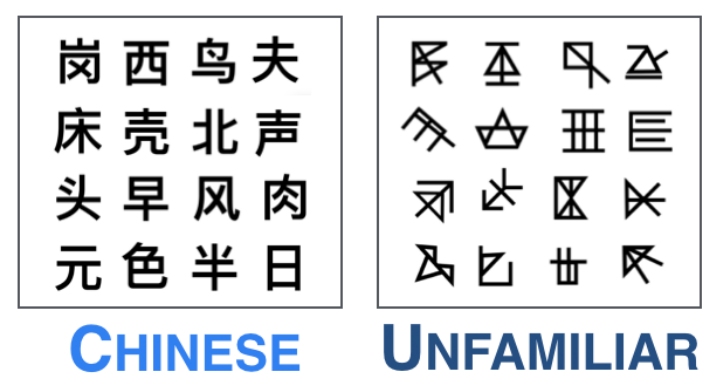

Figure 8. Experiment 3 stimuli. All participants saw both the CHINESE and UNFAMILIAR items in two separate statistical learning and two separate perceptual fluency tasks.

population pool, it was necessary to have an experiment that could be completed in one session; use of this pool was important as it contained many native Chinese speakers who matched the nonChinese speakers on age and education level. Second, the important comparisons for this experiment were between groups rather than between tasks, which necessitated a different structure.

We therefore presented each participant with two statistical learning tasks, one with each kind of stimuli (UNFAMILIAR and CHINESE), with the order randomised. Each person then also completed two perceptual fluency tasks with the same stimuli, in the same order as in the statistical learning tasks. Thus, a participant who saw the CHINESE stimuli the the first SL task would see them in the first PF task as well. This ensured that nobody performed two adjacent tasks with the same stimuli. The statistical learning and perceptual fluency tasks themselves were identical to those in Experiment 1.

\section{Experiment 3: Results}

The purpose of this experiment was to further explore whether stimulus familiarity or complexity drove the effects in the previous experiment. If, as hypothesised, it is due to familiarity, that yields several (pre-registered) predictions. First, it suggests an interaction between speaker and stimulus on statistical learning tasks, such that Chinese speakers will perform better on the CHINESE stimuli than in any other case. Second, it suggests the same interaction on the perceptual fluency task. And third, it suggests that perceptual fluency should be correlated with statistical learning, especially on the same stimuli. We consider each prediction in turn.

\section{Statistical learning}

As in the previous experiments, accuracy on the statistical learning task (SLscore) spans the range of individual differences, ranging between 19.0\% and 100\% (mean: 48.9\%). Performance was significantly above the chance level of $40 \%(t(353)=11.8, p<0.0001, d=3.43)$ and there was once again a high correlation between the item-level accuracy of the participants of Siegelman et al. (2017) and ours on both the first statistical learning task $(r=0.64, p<0.0001)$ and the second $(r=0.75, p<0.0001)$.

If familiarity rather than complexity drives the effects we have seen so far, we should expect that there should be an improvement in statistical learning performance (as measured by SLscore) only for those participants who saw familiar stimuli: the Chinese speakers shown Chinese characters. That is, we should expect an interaction between participant and stimulus. Conversely, if 
stimulus complexity were driving the effect, we should expect no interaction.

The results, shown in Figure 9, support the familiarity hypothesis: Chinese speakers show improved performance on Chinese stimuli, and the interaction is significant (stimulus: $F(1,704)=$ 9.1698, $p=0.0026$; participant: $F(1,704)=0.0062, p=0.9375$; interaction: $F(1,704)=$ $29.4098, p<0.0001)$. Post-hoc t-tests reveal that, as expected, English speakers performed similarly on the CHINESE and UNFAMILIAR stimuli $(t(461.9)=0.758, p=0.449)$ while Chinese speakers performed significantly worse on the UNFAMILIAR stimuli than they did on the CHINESE stimuli $(t(195.1)=-5.804, p<0.0001)$. These results indicate that stimulus familiarity, not absolute complexity, drives these differences in statistical learning.

Statistical learning by participant group

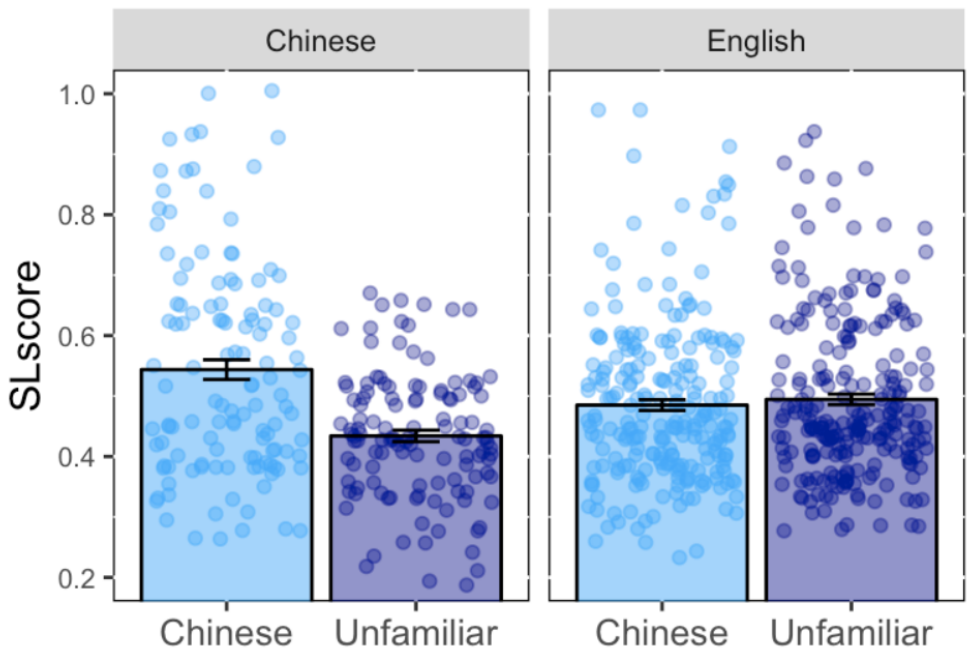

Figure 9. Experiment 3 statistical learning. As predicted by the familiarity hypothesis, there was an interaction between participant and stimulus type: Chinese speakers (left panel) showed higher statistical learning performance on the CHINESE stimuli than did English speakers.

\section{Perceptual fluency}

By a similar logic as above, if familiarity underlies perceptual fluency, we should expect an interaction between participant and stimuli on PFscore as well. ${ }^{5}$ As Figure 9 shows, this is indeed what we found: Chinese speakers performed better (with a lower PFscore) on the Chinese stimuli than anybody did on any of the other stimuli (stimulus: $F(1,698)=30.885, p<0.0001$; participant: $F(1,698)=30.066, p<0.0001$; interaction: $F(1,698)=38.7714, p<0.0001)$.

We can also investigate whether perceptual fluency is associated with statistical learning. If familiarity has something to do with this, one would expect that the correlation between PFscore and SLscore would be higher when the stimuli were the same (both CHINESE or both UNFAMILIAR) than when they were different (i.e., PF for CHINESE and SL for UNFAMILIAR or PF for UNFAMILIAR and SL for CHINESE). This was indeed the case (same: $r=-0.2, p<0.0001$; different:

\footnotetext{
${ }^{5}$ As pre-registered and as in Experiment 1, we removed the six PFscore values that were more than 4SD from the mean. Including them does not change any of our qualitative results.
} 
$r=-0.04, p=0.45)$. The difference between these correlations was significant using a Fisher r-to-z transformation $(z=-2.1, p=0.036)$.

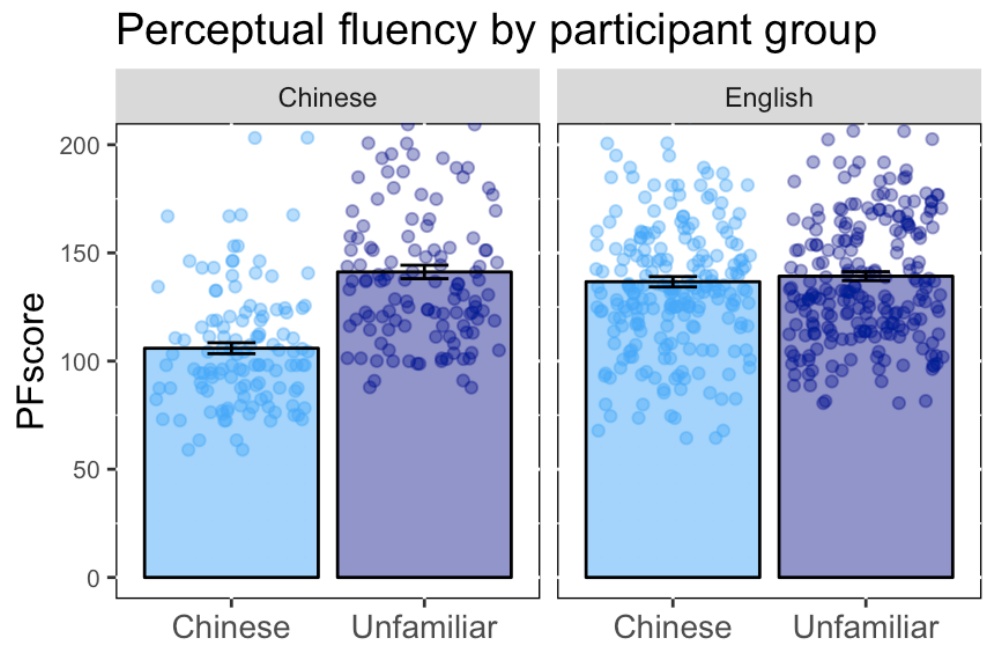

Figure 10. Experiment 3 perceptual fluency. As predicted by the familiarity hypothesis, there was an interaction between participant and stimulus type: Chinese speakers (left panel) showed improved perceptual fluency (as reflected by a lower PFscore on the CHINESE stimuli than did English speakers.

\section{General Discussion}

This work investigated the degree to which perceptual fluency influences statistical learning, and to what extent perceptual fluency reflects stimulus familiarity vs complexity. Recognising that SL relies on basic memory processes (Frank et al., 2010; Frost et al., 2019; Isbilen et al., 2020), we hypothesised that participants would learn the same statistical distribution better when they found items to be easier to perceptually distinguish, parse, and remember. This hypothesis was uniformly supported across three experiments. In Experiment 1, while participants performed significantly above chance on both SL tasks, they performed significantly better on the task containing overlearned items, even though the modality was the same in both cases. This suggests that the ease with which statistical distributions are learned is moderated not just by a person's modality-specific level of perceptual fluency, but also by their familiarity with the set of items. These effects were also observable at the individual level: test-retest correlations were higher when the two tasks used items that were equally familiar as each other, even though none of the specific individual items were repeated. Additionally, individual performance on both SL tasks was significantly associated with performance on a novel independent measure of perceptual fluency. In Experiment 2, we found that SL performance was not improved when the stimuli were simpler but still unfamiliar. Finally, in Experiment 3 we held stimulus complexity constant and varied participant experience with the experimental material, and once again found that participants performed best on the stimuli with which they were most familiar.

Overall, these data identify a fundamental role for perceptual fluency in statistical learning and demonstrate that perceptual fluency not only varies significantly between individuals but is also moderated by familiarity. Our findings are consistent with the overwhelming evidence that 
frequency matters in almost every aspect of cognition, from language to decision making to memory (e.g., Hasher \& Zacks, 1984; Sedlmeier \& Betsch, 2002; Gries \& Divjak, 2012; Baayen, Milin, \& Ramscar, 2016). Given the ubiquity of frequency effects, it is perhaps not particularly surprising if statistical learning is also improved when the items involved are highly familiar, having occurring frequently in prior experience.

Our work thus suggests an important modification to existing theories of statistical learning (e.g., Frost et al., 2015). Rather than postulating that individual variation in the efficiency of perceptual coding is entirely based on modality or timing, our results indicate that familiarity plays an important moderating role. We do not suggest that there are no modality-specific individual differences in perceptual fluency, but we do suggest that familiarity may be far more important than has previously been recognised; indeed, it is possible that some of the variation that has historically been attributed to modality differences may reflect familiarity differences instead.

Whether familiarity would have the exact same effect on statistical learning in the auditory modality remains an open question, as to our knowledge comparable studies to ours do not exist. However, there is evidence in the auditory domain that high frequency and therefore more familiar items can play facilitative roles in speech segmentation in both infants (Bortfield, Morgan, Golinkoff, \& Rathbun, 2005; Monaghan \& Christiansen, 2010) and adults (Frost, Monaghan, \& Christiansen, 2016). Other research has shown an important role for high frequency marker words in artificial grammar learning (e.g., Valian \& Coulson, 1988). In these studies the high frequency familiar items serve as cues to higher-level structure, and their designs are interesting because they mimic asymmetrical (e.g., Zipfian) frequency distributions present in language. An auditory analogue of our current study would show something even more fundamental, suggesting that familiarity with a lower-level features of language (e.g., a syllable or phoneme inventory) might provide an important basis for the statistical learning of higher level forms (e.g., words).

We have interpreted our results as reflecting the importance of familiarity, but one might question to what extent they reflect verbalisability instead: perhaps people performed better for familiar stimuli not because they were familiar per se, but because they had names and were thus easier to verbalise. This possibility is consistent with research suggesting that statistical learning is improved for stimuli that are easy to encode verbally (Conway, Karpicke, \& Pisoni, 2007). It is probable that verbalisability does play some role in our results, and it is impossible to rule it out entirely: any stimulus that is familiar enough to improve statistical learning will also almost certainly become associated with a label or mental shorthand. That said, several considerations suggest that our results are not entirely due to an improved ability to verbalise familiar stimuli. First, verbaliseability cannot explain the significant correlations found between performance on the PF and SL tasks. The PF task was a stimulus-specific task which had no component of statistical learning at all, and occurred too rapidly (on the order of $120-130 \mathrm{~ms}$ ) for verbalisation to be a factor in performance. For similar reasons, verbaliseability cannot explain the improved PFscore Chinese people had for CHINESE stimuli. Second, verbaliseability cannot explain why in Experiment 3 the Chinese participants actually performed worse in the SL task on the UNFAMILIAR stimuli than the English participants did $(t(298.7)=-4.7, p<0.0001)$; the UNFAMILIAR should be equally unverbaliseable for both populations. This result might instead indicate a form of perceptual interference, with UNFAMILIAR stimuli being harder for Chinese people to encode because of their similarity to the highly familiar Chinese characters. This interpretation is speculative, but regardless, it does suggest that at least part of the performance differential in the statistical learning task reflects familiarity rather than verbaliseability. 
Our work is complementary to entrenchment accounts of statistical learning, which suggest that the prior experience of transition probabilities can interfere with the learning of novel transition probabilities over the same items (Endress \& Mehler, 2009; Siegelman et al., 2018; Perruchet \& Poulin-Charronnat, 2012). The results are an interesting contrast to these cases, because our focus has been on the experience with the stimuli themselves (rather than the TPs) and because (other than the Chinese participants with UNFAMILIAR stimuli) we found a facilitative rather than disadvantageous affect of prior experience. The facilitative effect makes sense given our focus on the stimuli themselves, since the relevant prior experience did not contradict what participants saw in experiment (whereas in previous research, the TPs in the experiment contradicted the learned TPs in the language). In our work, people's previous experience with letters and characters may have made those stimuli easier to rapidly encode, thus allowing more processing resources to be devoted to the statistical learning component of the task. The effect of stimulus familiarity seems to have been substantial enough to override any interference caused by any pre-existing TPs (letter-letter associations) that participants may have had. Although our stimuli were controlled such that no participant saw the exact same combinations of items, literate participants are likely to have implicit expectations of relative letter locations, which could have but did not influence pattern detection.

Overall, our results support the suggestion that, rather than being a purely abstract and domain-general process, SL is a multicomponential process grounded in basic cognition (Arciuli, 2017; Siegelman et al., 2017; Frost et al., 2019; Growns, Siegelman, \& Martire, 2020). Indeed, we found that within-domain variation in perceptual fluency (both within and between individuals) plays a significant role in learning. These data are important, in the first instance, because they broaden the scope of variables that influence statistical learning. Frost et al. (2019) argued that, while SL is likely implicated in most domains of cognition, research on the topic has pursued a more isolationist strategy, and as such its promise as an explanatory concept has not been realised. Our finding that perceptual fluency influences SL naturally lend themselves to Frost et al. (2019)'s call for domain integration because they ground learning in basic memory and perceptual processes.

A natural question, which we cannot fully answer here, is how perceptual fluency and SL interact. On the one hand, it is clear that stimulus encoding is a pre-condition for learning probability distributions across items ((Bogaerts et al., 2016); however, if SL plays a fundamental role in learning, then the relationship should be bidirectional, such that perceptual features may be sharpened by predictable patterns over time. By way of example, consider how the two might interact in reading acquisition. Once a child learns individual letters they begin to coordinate those letters into words, which in languages like English is difficult because of the one-to-many grapheme-to-phoneme mappings. Initially this process is laboured for children, whose strategies vacillate between whole form memorization and phoneme-by-phoneme pronunciation. However, an account where SL is important for reading (Arciuli, 2018) predicts that, as the learner identifies regularities in the visual input, orthographic representations will be strengthened, thus completing a virtuous circle.

This work suggests part of a solution to the question of exactly what statistical learning is and how it relates to other cognitive skills. If statistical learning is mediated by perceptual fluency, and that in turn is affected by familiarity and prior experience, then we should expect a degree of modality-specific effects on learning, which is consistent with the bulk of past research. As such, we take a further step away from the conceptualisation of performance on SL tasks as measuring domain-general computations over amodal abstract representations. These findings also suggest that one way to improve statistical learning (and any learning that relies on it) is simply to increase exposure to the stimuli involved. We are particularly excited for the potential of this possibility, 
since SL underlies learning in so many different domains and increased exposure is in many cases a relatively easy and cheap intervention. Much remains to be done, but our work opens the door to a variety of advances, both theoretical and applied.

\section{Acknowledgments}

Research costs for AP were funded through ARC grants DP150103280 and DP180103600. We would also like to thank Jing Qian and Rachel Yam for their intellectual contributions to the experimental design, and Rebecca Frost for helpful feedback.

\section{References}

Alvarez, G., \& Cavanagh, P. (2004). Capacity of visual short-term memory is set both by visual information load and by number of objects. Psychological Science, 15, 106-111.

Arciuli, J. (2017). The multi-component nature of statistical learning. Philosophical Transactions of the Royal Society B, 372(1711), 1-9.

Arciuli, J. (2018). Reading as statistical learning. Language, Speech, and Hearing Services in Schools, 49, 634-643.

Arciuli, J., \& Simpson, I. (2011). Statistical learning in typically developing children: The role of age and speed of stimulus presentation. Developmental Science, 14, 464-473.

Baayen, H., Milin, P., \& Ramscar, M. (2016). Frequency in lexical processing. Aphasiology, 30(11), 11741220.

Bogaerts, L., Siegelman, N., \& Frost, R. (2016). Splitting the variance of statistical learning performance: A parametric investigation of exposure duration and transitional probabilities. Psychonomic Bulletin and Review, 23, 1250-1256.

Bortfield, H., Morgan, J., Golinkoff, R., \& Rathbun, K. (2005). Mommy and me: Familiar names help babies into speech segmentation. Psychological Science, 16, 298-304.

Brady, T., \& Oliva, A. (2008). Statistical learning using real-world scenes: Extracting categorical regularities without conscious intent. Psychological Science, 19(7), 678-685.

Buchsbaum, D., Griffiths, T., Plunkett, D., Gopnik, A., \& Baldwin, D. (2015). Inferring action structure and causal relationships in continuous sequences of human action. Cognitive Psychology, 76, 30-77.

Bulf, H., Johnson, S., \& Valenza, E. (2011). Visual statistical learning in the newborn infant. Cognition, $121(1), 127-132$.

Christiansen, M. (2019). Implicit statistical learning: A tale of two theories. Topics in Cognitive Sciences, $11,468-481$.

Conway, C., \& Christiansen, M. (2006). Statistical learning within and between modalities: Pitting abstract against stimulus-specific representations. Psychological Science, 17(10), 905-912.

Conway, C., Karpicke, J., \& Pisoni, D. (2007). Contribution of implicit sequence learning to spoken language processing: Some preliminary findings with hearing adults. Journal of Deaf Studies and Deaf Education, 12(3), 317-334.

Endress, A., \& Mehler, J. (2009). The surprising power of statistical learning: When fragment knowledge leads to false memories of unheard words. Journal of Memory and Language, 60, 351-367.

Fiser, J., \& Aslin, R. (2002). Statistical learning of new visual feature combinations by infants. Proceedings of the National Academy of Sciences, 99(24), 15822-15826.

Frank, M. C., Goldwater, S., Griffiths, T. L., \& Tenenbaum, J. B. (2010). Modeling human performance in human speech segmentation. Cognition, 117, 107-125.

Frost, R., Armstrong, B., \& Christiansen, M. (2019). Statistical learning research: A critical review and possible directions. Psychological Bulletin, 145, 1128-1153.

Frost, R., Armstrong, B., Siegelman, N., \& Christiansen, M. (2015). Domain generality versus modality specificity: The paradox of statistical learning. Trends in Cognitive Sciences, 19(3), 117-125. 
Frost, R., Monaghan, P., \& Christiansen, M. (2016). Using statistics to learn words and grammatical categories: How high frequency words assist language acquisition. In A. Papafragou, D. Mirman, \& J. Trueswell (Eds.), Proceedings of the 38th Annual Conference of the Cognitive Science Society (p. 81-86). Austin, TX: Cognitive Science Society.

Gebhart, A., Newport, E., \& Aslin, R. (2009). Statistical learning of adjacent and nonadjacent dependencies among nonlinguistic sounds. Psychonomic Bulletin and Review, 16, 486-490.

Gries, S., \& Divjak, D. (Eds.). (2012). Frequency effects in language learning and processing. De Gruyter Mouton.

Growns, B., Siegelman, N., \& Martire, K. (2020). The multi-faceted nature of visual statistical learning: Individual differences in learning conditional and distributional regularities across time and space. Psychonomic Bulletin \& Review.

Hasher, L., \& Zacks, R. (1984). Automatic processing of fundamental information: The case of frequency of occurrence. American Psychologist, 39(12), 1372-1388.

Hauser, M., Newport, E., \& Aslin, R. (2001). Segmentation of the speech stream in a nonhuman primate: Statistical learning in cotton-top tamarins. Cognition, 78, B53-B64.

Hofmeister, P. (2011). Representational complexity and memory retrieval in language comprehension. Language and Cognitive Processes, 26(3), 376-405.

Isbilen, E., McCauley, S., Kidd, E., \& Christiansen, M. (2017). Testing statistical learning implicitly: A novel chunk-based measure of statistical learning. In G. Gunzelmann, A. Howes, T. Tenbrink, \& E. Davelaar (Eds.), Proceedings of the 39th Annual Conference of the Cognitive Science Society (p. 564-569). Austin, TX: Cognitive Science Society.

Isbilen, E., McCauley, S., Kidd, E., \& Christiansen, M. (2020). Statistically-induced chunking recall: A memory-based approach to statistical learning. Cognitive Science, 44.

Jackson, M., \& Raymond, J. (2008). Familiarity enhances visual working memory for faces. Journal of Experimental Psychology: Human Perception and Performance, 34(3), 556.

Kaufman, S., DeYoung, C., Gray, J., Jiménez, L., Brown, J., \& Mackintosh, N. (2010). Implicit learning as an ability. Cognition, 116, 321-340.

Kirkham, N., Slemmer, J., \& Johnson, S. (2002). Visual statistical learning in infancy: Evidence for a domain general learning mechanism. Cognition, 83, B35-B42.

Krogh, L., Vlach, H., \& Johnson, S. (2013). Statistical learning across development: Flexible yet constrained. Frontiers in Psychology, 3, 598.

Luck, S., \& Vogel, E. (1997). The capacity of visual working memory for features and conjunctions. Nature, 390(6657), 279-281.

Monaghan, P., \& Christiansen, M. (2010). Words in puddles of sound: Modelling psycholinguistic effects in language acquisition. Journal of Child Language, 37, 545-564.

O’Connor, T., \& Burns, N. (2003). Inspection time and general speed of processing. Personality and Individual Differences, 35(3), 713-724.

Perruchet, P., \& Poulin-Charronnat, B. (2012). Beyond transitional probability computations: Extracting word-like units when only statistical information is available. Journal of Memory and Language, 66(4), 807-818.

Saffran, J., Aslin, R., \& Newport, E. (1996). Statistical learning by 8-month-old-infants. Science, 274(5294), 1926-1928.

Sedlmeier, P., \& Betsch, T. (Eds.). (2002). Etc: Frequency processing and cognition. New York, NY: Oxford University Press.

Siegelman, N., Bogaerts, L., Elazar, A., Arciuli, J., \& Frost, R. (2018). Linguistic entrenchment: Prior knowledge impacts statistical learning performance. Cognition, 177, 198-213.

Siegelman, N., Bogaerts, L., \& Frost, R. (2017). Measuring individual differences in statistical learning: Current pitfalls and possible solutions. Behavior Research methods, 49(2), 418-432.

Siegelman, N., \& Frost, R. (2015). Statistical learning as an individual ability: Theoretical perspectives and empirical evidence. Journal of Memory and Language, 81, 105-120. 
Teinonen, T., Fellman, V., Näätänen, R., Alku, P., \& Huotilainen, M. (2009). Statistical language learning in neonates revealed by event-related brain potentials. BMC Neuroscience, 10, 127-132.

Turk-Browne, N., Junge, J., \& Scholl, B. (2005). The automaticity of visual statistical learning. Journal of Experimental Psychology: General, 134(4), 552-564.

Valian, V., \& Coulson, S. (1988). Anchor points in language learning: The role of marker frequency. Journal of Memory and Language, 27, 71-86.

Xie, W., \& Zhang, W. (2017). Familiarity increases the number of remembered pokemon in visual short-term memory. Memory and Cognition, 45(4), 677-689. 


\section{Appendix A: Further analyses for Experiments 1 and 2}
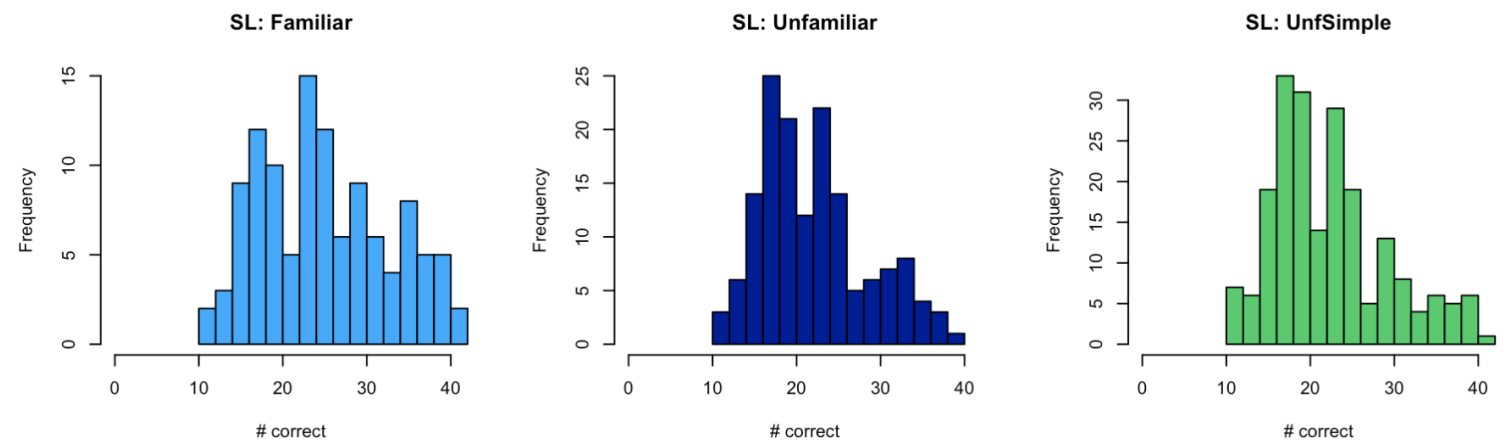

Figure 11. Histograms of accuracy on the SL tasks. Each histogram plots the individual overall SLscore for each participant, broken down by condition. It is evident that there is a wide range of individual variation within each condition, and that the histograms appear approximately normal and similar in character to those reported in Siegelman et al (2017).
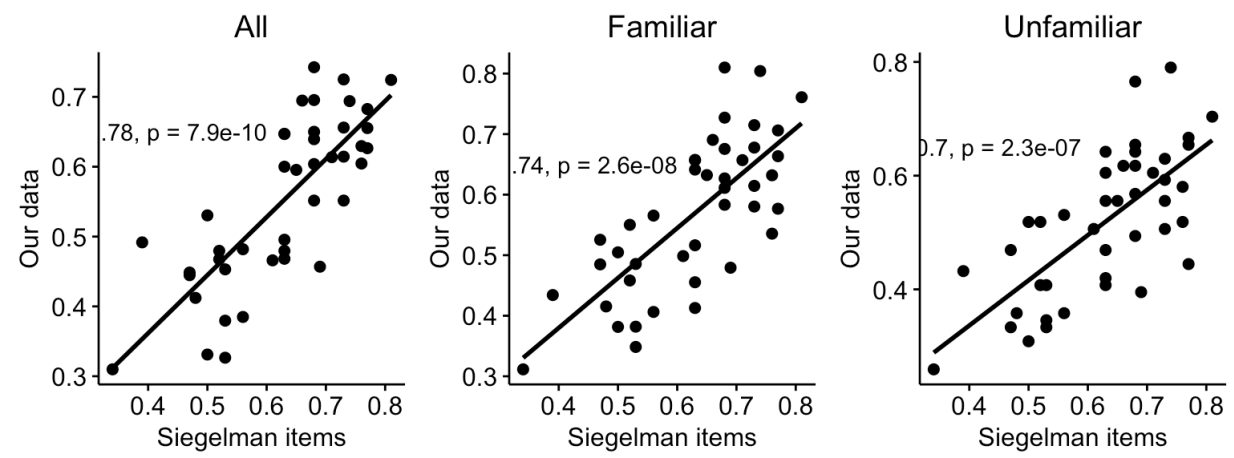

Figure 12. Item-level correlations with data from Siegelman et al (2017). Each plot shows the correlation of performance on each of the 42 test items in the SL task in our study and that of Siegelman et al (2017). Correlations are strong and highly significant whether calculated over all stimuli (left), just the FAMILIAR ones (middle), or just the UNFAMILIAR ones (right). This demonstrates that our participants were approaching the task similarly to theirs. 

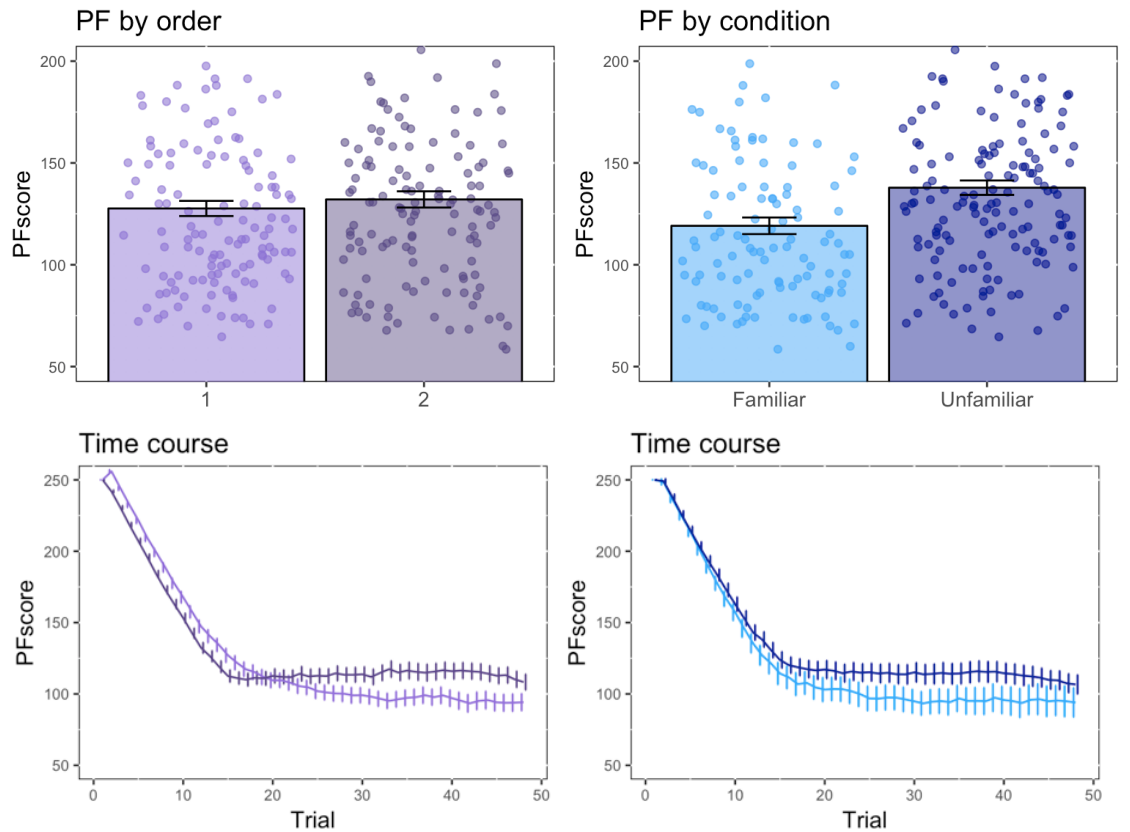

Figure 13. Performance in perceptual fluency task. The panels on the left show PFscore as a function of whether the PFtask was the first or the second the participant did. The time course (bottom) reveals that there is a small effect of both learning (improved performance initially on the second task) and fatigue (improved performance at the end of the first task). However, as the bar graph at the top reveals, these effects cancel each other out and there is no significant difference by order of the task. Conversely, the panels on the right show that there is consistently better performance with the FAMILIAR stimuli than with the UNFAMILIAR stimuli.
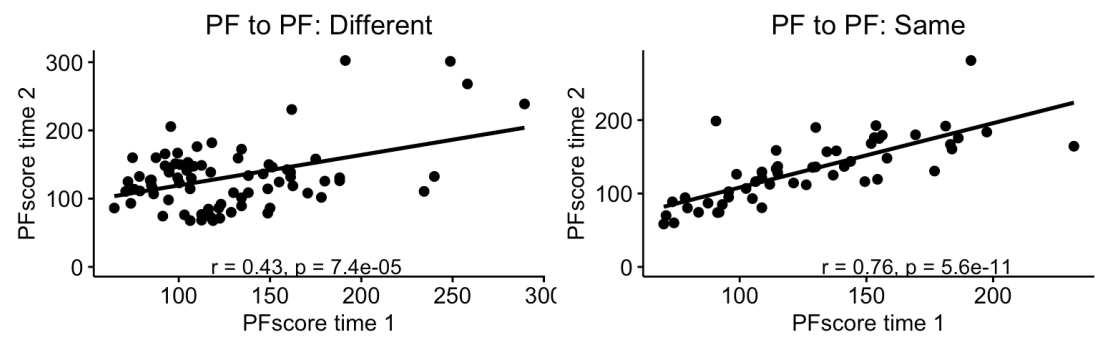

Figure 14. Correlations between PFscores. Each plot shows the correlation of performance on the two PF tasks each person took, broken down by whether the stimuli were from the same set (both UNFAMILIAR or both FAMILIAR). Correlations are significant for both, but higher when the stimuli were from the same set. This suggests that the PF tasks reflects aspects of both stimulus-specific encoding efficiency as well as more general speed of encoding or processing. 


\section{Appendix B: Stimuli for Experiment 3}

1. 他们

(a) metal door (b) they (c) government (d) her

2. 球

(a) spread (b) beg (c) play (d) ball

3. 没有

(a) yes (b) present (c) not (d) sister

4. 红

(a) red (b) blue (c) green (d) yellow

\begin{tabular}{|l|l|l|}
\hline Character & Pronunciation & Meaning \\
\hline 日 & rì & day/ date \\
\hline 夫 & fū & husband \\
\hline 元 & yuán & dollar \\
\hline 风 & fēng & wind \\
\hline 头 & tóu & head \\
\hline 半 & bàn & half \\
\hline 皮 & pí & skin \\
\hline 西 & xī & East \\
\hline 色 & sè & colour \\
\hline 早 & zăo & morning/ early \\
\hline 肉 & ròu & meat/ flesh \\
\hline 声 & shēng & sound \\
\hline 壳 & ké & shell \\
\hline
\end{tabular}

Figure 15. Stimuli used in Experiment 3. On the left are the four test questions used to evaluate Chinese proficiency; correct answers are italicised. The table on the right shows all 16 Chinese characters along with their pronunciation and meaning. All corresponded to nouns and varied widely in initial phoneme and tone. 


\section{Appendix C: Additional analyses for Experiment 3}

\section{Histograms of SL scores}

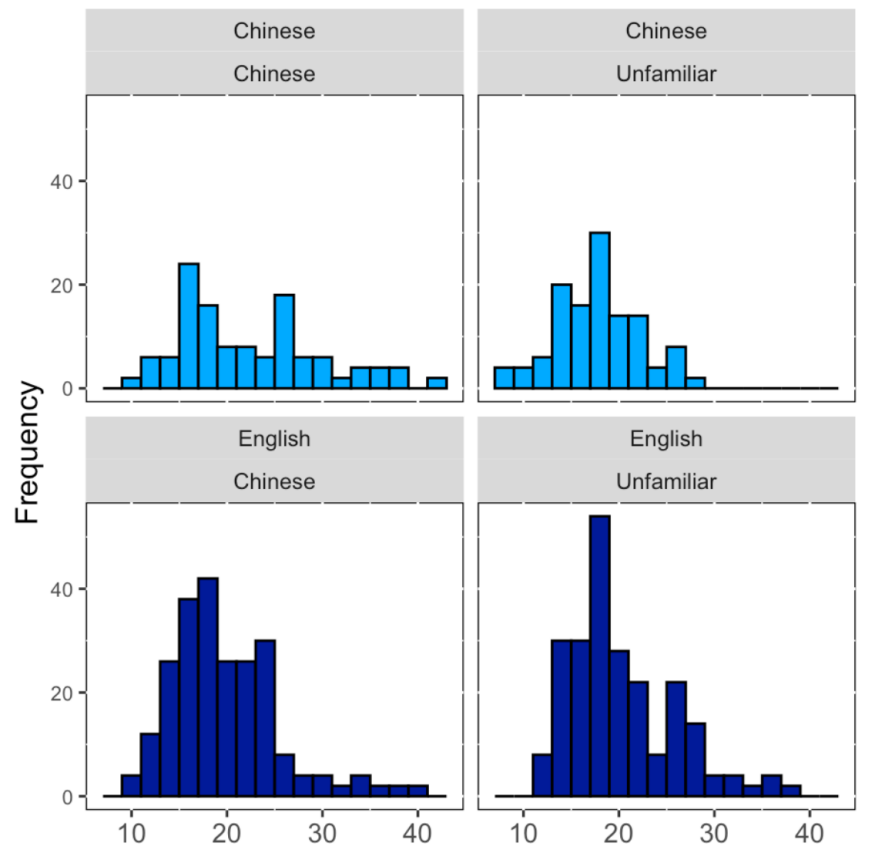

Figure 16. Histograms of accuracy on the SL tasks. Each histogram plots the individual overall SLscore for each participant, broken down by condition. As before, there is a wide range of individual variation within each condition, and the histograms appear approximately normal and similar in character to those reported in Siegelman et al (2017). 

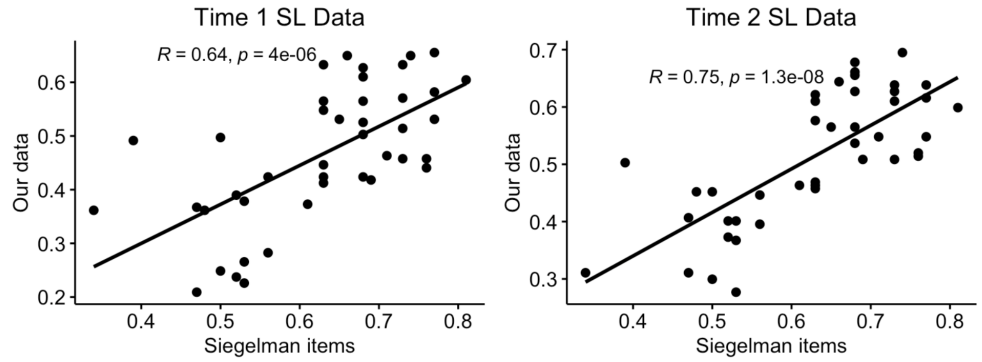

Figure 17. Item-level correlations with data from Siegelman et al (2017). Each plot shows the correlation of performance on each of the 42 test items in the SL task in our study and that of Siegelman et al (2017), broken down by whether it was the first or the second task. As before, correlations are strong and highly significant, which this demonstrates that our participants were approaching the task similarly to theirs.

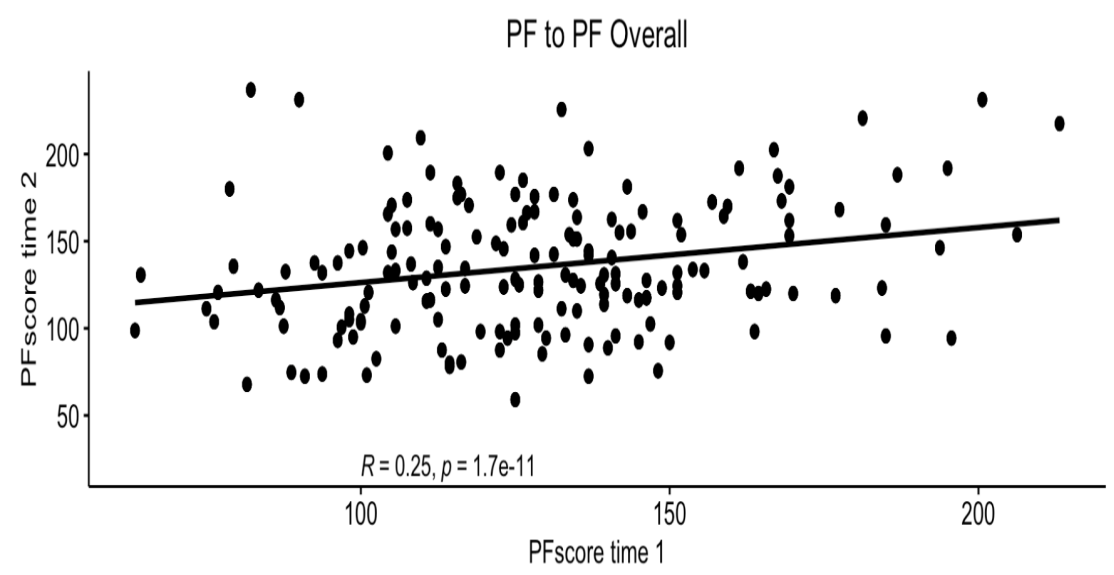

Figure 18. Correlations between PFscores. Correlation of performance on the two PF tasks each person took. As before, the correlation is positive and significant. 